Artículo

\title{
Efectividad de extractos biológicos y químicos comerciales para el control de nematodos en cafeto en Chiapas
}

\author{
Melchor Cepeda Siller \\ Yisa María Ochoa Fuentes \\ Ernesto Cerna Chávez \\ Fabiola Garrido Cruz \\ Aideé González Ruíz \\ Agustín Hernández Juárez ${ }^{\S}$
}

Universidad Autónoma Agraria Antonio Narro-Departamento de Parasitología. Calzada Antonio Narro núm. 1923. Buenavista, Saltillo, Coahuila, México. CP. 25315. Tel. 8444110326. (melchoresraza2010@hotmail.com; yisa8a@yahoo.com; jabaly1@yahoo.com; fabygarrido@hotmail.com; daryna_85@hotmail.com).

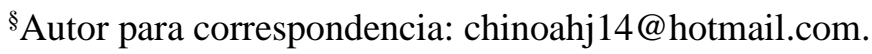

\section{Resumen}

Los nematodos fitoparásitos constituyen uno de los principales patógenos que afectan el cultivo del cafeto Coffea arabica L., causando reducciones en el rendimiento entre 15 y $60 \%$. El objetivo de esta investigación fue evaluar la efectividad de los extractos biológicos del nematicida Nemaxxion XT Plus, para el control de los nematodos Meloidogyne incognita y Pratylenchus sp., asociados al cultivo del café. Se consideraron cinco tratamientos con cinco repeticiones bajo un diseño en bloques completos al azar: Nemaxxion XT Plus (2 $\left.\mathrm{L} \mathrm{ha}^{-1}, 4 \mathrm{~L} \mathrm{ha}^{-1}, 6 \mathrm{~L} \mathrm{ha}^{-1}\right)$, Nemacur ${ }^{\circledR}$ $400 \mathrm{CE}$ y testigo. Se realizó el conteo de las poblaciones de nematodos en $100 \mathrm{~g}$ de suelo antes de la aplicación de los tratamientos y a los 120 días de instalado el experimento. Para el conteo, los nematodos se extrajeron por el método de embudo de Baerman. Para M. incognita, se contabilizó el número de juveniles de segundo estadio, machos y hembras adultos y para Pratylenchus sp., se contabilizaron los machos y hembras adultos filiformes. La evaluación demostró la efectividad de los tratamientos para el control de las poblaciones de nematodos, estos tratamientos presentaron una reducción considerable de las poblaciones, con una sobrevivencia en promedio de 1.2-3.4 nematodos de M. incognita y 2.2-4.4 nematodos de Pratylenchus sp., de estos, Nemaxxion XT Plus a $6 \mathrm{~L} \mathrm{ha}^{-1}$ presentó la mayor reducción poblacional y eficiencia sobre las dos poblaciones, con 99.08 y $92.87 \%$ respectivamente, presentándose como alternativa para una agricultura más sustentable.

Palabras clave: Meloidogyne incognita, Pratylenchus sp., nematodo agallador, nematodo lesionador.

Recibido: junio de 2020

Aceptado: agosto de 2020 


\section{Introducción}

El café o cafeto (Coffea spp.), es una planta de la familia Rubiaceae y es originaria de Etiopía. Fue cultivado por primera vez en la provincia de Kaffa en Etiopia a mediados del siglo XII, desde donde se extendió a través del comercio a medio oriente y a partir del siglo XV se introdujo a Europa, donde su consumo se generalizó durante los siguientes 200 años (Pérez y Díaz, 2000).

En la actualidad, son conocidas más de cien especies de Coffea, de las cuales Coffea arabica L. y Coffea canephora L., son las dos especies cultivadas más importantes, la primera con una aportación de 58\% de la producción mundial y la segunda con 42\% (ICO, 2017). Para 2014 los principales productores de cafeto verde en el mundo fueron, Brasil con $2804070 \mathrm{t}$, Vietnam con 1406469 t, Colombia con 728400 t, Indonesia con 643900 t y Etiopia con 419980 t (FAO, 2017).

En México, Coffea arabica L. ocupa más de $97 \%$ de la superficie cafetalera y el cafeto robusta Coffea canephora L. (Var. Robusta) en menor medida se ubica en el resto de la superficie, esta última dirigida casi exclusivamente al sector de los cafés solubles. Actualmente se contabilizan cerca de 120 variedades del café arábica; destacando las variedades Criollo (Typica), Bourbón, Catimores, Caturra (rojo y amarillo), Colombia, Costa rica, Garnica, Maragogipe, Mundo novo, Oro azteca, Pacamara y Pluma hidalgo (AMECAFÉ, 2016).

En 2015, en 16 estados de la república mexicana se produjeron 102625198 t de cafeto, en una superficie de 73429103 ha, distribuidas en las zonas serranas que atraviesan el país, con un valor de la producción de $\$ 5340761$ 16. En este sentido, los principales productores con $64.2 \%$ de la producción nacional, son los estados de Chiapas y Veracruz con 38305962 (37.3\%) y 27605471 (26.9\%) ton respectivamente (SAGARPA-SIAP, 2017).

La presencia de plagas es de gran importancia en el cultivo del cafeto, principalmente por su alta incidencia o por el nivel de daño que ocasionan. Los nematodos fitoparásitos constituyen uno de los principales patógenos limitantes que afectan el cultivo del cafeto, particularmente la especie Coffea arabica que es susceptible a los nematodos; capaces de causar reducciones en el rendimiento que oscilan entre 15-60\% (Campos y Villain, 2005; ANACAFÉ 2017).

Numerosas especies de nematodos han sido señaladas en asociación con este cultivo; sin embargo, las especies de los géneros Pratylenchus Filipjev (Tylenchida: Pratylenchidae) y Meloidogyne Goeldi (Tylenchida: Heteroderidae) son los más ampliamente distribuidos y los de mayor importancia económica, con las mayores pérdidas en el cultivo; los primeros con pérdidas en el rendimiento de $29-78 \%$ y los últimos, pérdidas en rendimiento de entre $10-15 \%$ o hasta $100 \%$ bajo determinadas condiciones (Inomoto et al., 1998; Oliveira et al., 1999; Campos y Villain, 2005; Carneiro y Cofcewicz, 2008; Villain, 2008; Souza y Bressan-Smith, 2008).

El problema fitosanitario con nematodos va en incremento, y las alternativas de control son pocas, principalmente se utilizan aplicaciones sistemáticas de compuestos químicos, por su efecto eficaz sobre las diferentes especies de nematodos fitoparásitos (Perry, 1998; Baños et al., 2010). No obstante, su empleo ha sido restringido por los efectos nocivos al ambiente y pueden llegar a ser muy tóxicos para la salud de productores y consumidores (Fe, 2002; Baños et al., 2010). 
Por otro lado, el costo, la residualidad y fitotoxicidad de los nematicidas para el cultivo, limitan su aplicación, por lo que es imprescindible buscar alternativas para combatir nematodos fitopatógenos, que afectan considerablemente la producción (Hernández et al., 2015).

Actualmente la agricultura demanda la reducción de productos químicos y un aumento de productos amigables con el medio ambiente y la salud humana; por esta razón, el control biorracional; a partir de extractos y organismos de control biológico, se presenta como alternativa muy prometedora, que permite el desarrollo de una agricultura más rentable y no contaminante del medio ambiente; motivo por el cual se plantea evaluar la efectividad biológica de Nemaxxion XT Plus, con base en extractos vegetales y componentes orgánicos para el control de los nematodos Meloidogyne incognita y Pratylenchus sp., asociados al cafeto en Motozintla, Chiapas.

\section{Materiales y métodos}

\section{Localización del experimento}

La investigación en campo se desarrolló en la Finca de producción comercial denominada Guadalupe Zajú, con el cultivo de cafeto Var. Catuaí en etapa de desarrollo de fruto, ubicadas en la región productora denominada 'El Soconusco', perteneciente al municipio de Motozintla, Estado de Chiapas, con un suelo migajón limoso y una elevación de $1000 \mathrm{msnm}$, con una temperatura promedio mínima de $22{ }^{\circ} \mathrm{C}$ y máxima de $29^{\circ} \mathrm{C}$ y una precipitación media mensual de $92 \mathrm{~mm}$.

La investigación correspondiente a laboratorio se llevó a cabo en el Laboratorio de Nematología del Departamento de Parasitología de la Universidad Autonoma Agraria Antonio Narro (UAAAN), en Saltillo, Coahuila, México.

\section{Muestreo inicial e identificación de nematodos}

Con la finalidad de reconocer la población de nematodos asociados al cultivo antes de la aplicación de tratamientos, se realizó un muestreo inicial en dos árboles de cafeto por unidad experimental, en el punto cardinal norte se tomó una sub muestra de suelo de $1 \mathrm{~kg}$ a una profundidad de $40 \mathrm{~cm}$ en cada árbol, las dos sub muestras se mezclaron y se tomó solo una muestra representativa de 1 $\mathrm{kg}$ de suelo, las 25 muestras compuestas fueron trasladadas al Laboratorio de Nematología; de cada muestra compuesta se procesaron $100 \mathrm{~g}$ para obtener los nematodos presentes, mediante el método de embudo de Baerman (Cepeda, 1995).

Los nematodos Meloidogyne sp. y Pratylenchus sp., fueron identificados a nivel de genero con base en las claves taxonómicas de Cepeda (2016). En hembras adultas del genero Meloidogyne se realizaron cortes perineales, siguiendo la técnica de Taylor y Netscher (1974) y para corroborar la especie se utilizaron las claves taxonómicas de Eisenback et al. (1981), coincidiendo con la especie Meloidogyne incognita.

\section{Aplicación de tratamientos}

Directamente en los árboles de cafeto se abrieron canales (zanjas) en forma circular en la zona de goteo del frutal, donde se colocaron los tratamientos a evaluar en contacto con el sistema radical, utilizando una mochila aspersor manual y posteriormente el canal fue cubierto con el mismo suelo. 
Se estableció un diseño en bloques completamente al azar (distancia de $2 \mathrm{~m}$ entre bloques) con cinco tratamientos y cinco repeticiones y cada repetición con una unidad experimental representada por dos árboles de cafeto de dos años, distribuidos a una distancia de plantación de $1 \mathrm{~m}$ (Cuadro 1$)$.

Cuadro 1. Tratamientos para evaluar la efectividad de control de nematodos asociados al cultivo del cafeto en Motozintla, Chiapas.

\begin{tabular}{clc}
\hline Tratamientos & Ingrediente activo(s) & ${\text { Dosis }\left(\mathrm{L} \mathrm{ha}^{-1}\right)}^{1}$ \\
\hline 1 & Extractos biológicos $^{1}$ & 2 \\
2 & Extractos biológicos $^{1}$ & 4 \\
3 & Extractos biológicos $^{1}$ & 6 \\
4 & Fenamifos $^{2}$ & 3 \\
5 & Agua $^{3}$ & 0 \\
\hline
\end{tabular}

${ }^{1}=$ productos proporcionados por GreenCorp Biorganiks de México SA de CV. Nemaxxion XT Plus= mezcla de extractos vegetales de cempasúchil $2 \%\left(2 \mathrm{~g} \mathrm{ml}^{-1}\right)$ Tagetes erecta $\mathrm{L}$. (Asteraceae), gobernadora $5 \%\left(5 \mathrm{~g} \mathrm{ml}^{-1}\right)$ Larrea tridentata L. (Sessé y Moc. Ex DC.) Coville (Zygophyllaceae), cáscara de nuez 5\% (5 g ml-1) Carya illinoinensis (Wangenh.) K. Koch (Juglandaceae), aceites esenciales de origen vegetal 10\% $\left(10 \mathrm{~g} \mathrm{ml}^{-1}\right)$ quitosan $2 \%\left(2 \mathrm{~g} \mathrm{ml}^{-1}\right)$, materia orgánica 5\% $\left(5 \mathrm{~g} \mathrm{ml}^{-1}\right)$, ácidos fulvicos de leonardita 30\% $\left(30 \mathrm{~g} \mathrm{ml}^{-1}\right)$ y emulsificantes y diluyentes $41 \%(41 \mathrm{~g}$ $\left.\mathrm{ml}^{-1}\right) ;{ }^{2}=$ Nemacur $^{\circledR} 400 \mathrm{CE}$, organofosforado sistémico, testigo químico (equivalente a $35 \mathrm{~g}$ de ia. $\mathrm{L}^{-1}$ ), ${ }^{3}=$ testigo absoluto.

Durante el desarrollo de la investigación, el nematicida Nemaxxion XT Plus se aplicó en tres ocasiones a partir del inicio de experimento en intervalos de 14 días entre aplicación y el nematicida Nemacur 400 CE se aplicó solo en una ocasión.

El manejo agronómico del cultivo durante el desarrollo del experimento se realizó con base a las prácticas típicas de la región, particularmente actividades de limpieza y manejo de enfermedades foliares; principalmente la roya del cafeto ocasionada por el hongo Hemileia vastatrix (Berkeley \& Broome) (Basidiomycota: Pucciniales), utilizando oxicloruro de cobre (fungicidas cúpricos).

\section{Muestreo final}

A los 120 días posteriores de la última aplicación de los tratamientos, en cada una de las unidades experimentales, en la zona de goteo del frutal, se realizó un muestreo final de igual forma que el muestreo inicial y las muestras fueron remitidas al laboratorio de Nematología, donde los nematodos fueron extraídos mediante el método de embudo de Baerman (Cepeda, 1995), identificados y contabilizados para obtener la población final.

Con los resultados se relacionó el índice de incremento de la población mediante la fórmula desarrollada por Seinhorst (1970): I= Pf/Pi. Donde: I= índice de incremento de la población, Pf= población final y $\mathrm{Pi}=$ población inicial.

Además, se determinó la eficiencia de los tratamientos mediante la fórmula propuesta por Henderson-Tilton (1955): $\mathrm{E}=(1-\mathrm{Pfa} / \mathrm{Pia} * \mathrm{Pib} / \mathrm{Pfb}) * 100$. Donde: $\mathrm{E}=$ eficiencia de los tratamientos; $\mathrm{Pia}=$ población inicial del tratamiento; $\mathrm{Pfa}=$ población final del tratamiento; $\mathrm{Pib}=$ población inicial del tratamiento testigo; $\mathrm{Pfb}=$ población final del tratamiento testigo. 
La evaluación de la actividad nematicida (reducción de la población) se obtuvo mediante la diferencia entre población inicial-población final y se analizó mediante un Anova con separación de medias de Tukey ( $p<0.05$ ), usando el software estadístico SAS 9.0 (SAS, Institute, 2002).

\section{Resultados y discusión}

En el Cuadro 2, se presenta la población al inicio de la investigación y la población final encontrada 120 días después de la aplicación de los tratamientos para el control de Meloidogyne spp. y Pratylenchus sp., obtenidos de suelo del cultivo de cafeto.

Cuadro 2. Media de la población inicial y población final de Meloidogyne incognita y Pratylenchus sp., en cada tratamiento, obtenidas de suelo de café.

\begin{tabular}{cccccc}
\hline \multirow{2}{*}{ Tratamientos } & \multicolumn{2}{c}{ Meloidogyne incognita } & & \multicolumn{2}{c}{ Pratylenchus sp. } \\
\cline { 2 - 3 } \cline { 5 - 6 } & Inicial & Final & & Inicial & Final \\
\hline 1 & 129.2 & 3.4 & & 35.2 & 4.4 \\
2 & 159 & 2.6 & & 34.6 & 4.2 \\
3 & 129.8 & 1.2 & & 32.8 & 2.2 \\
4 & 166.2 & 2.8 & & 29.6 & 3.6 \\
5 & 140.2 & 121.4 & & 44.2 & 57.2 \\
\hline
\end{tabular}

Se observa una reducción significativa de la población de nematodos por efecto de la aplicación de los tratamientos de control; mientras que el tratamiento testigo presentó un incremento de la población en Pratylenchus; no obstante, en $M$. incognita, el testigo presento una reducción de $12.84 \%$, con respecto a la población de inicio.

El Cuadro 3 muestra la reducción de la población de $M$. incognita y Pratylenchus sp., en el cual se observa que los tratamientos a base de extractos vegetales y componentes orgánicos presentan un control significativo $(p<0.05)$ sobre los diferentes nematodos del cultivo de café.

Cuadro 3. Reducción de las poblaciones de Meloidogyne incognita y Pratylenchus sp., después de la aplicación de tratamientos.

\begin{tabular}{clccc}
\hline Tratamientos & \multicolumn{3}{c}{ Reducción de la población de nematodos (\%) } \\
\cline { 2 - 5 } & 97.31 & $\mathrm{a}$ & 87.61 & Pratylenchus $\mathrm{sp}$. \\
\hline 1 & 98.38 & $\mathrm{a}$ & 87.47 & $\mathrm{a}$ \\
2 & 99.08 & $\mathrm{a}$ & 92.87 & $\mathrm{a}$ \\
3 & 98.28 & $\mathrm{a}$ & 87.83 & $\mathrm{a}$ \\
4 & 12.84 & $\mathrm{~b}$ & 0 & $\mathrm{~b}$ \\
5 & & & 0 & \\
\hline
\end{tabular}

Reducción de la población en la misma columna con la misma letra, no son significativamente diferentes (Tukey <0.05).

Entre los tratamientos de prueba (1-4) para el control de $M$. incognita., no se encontraron diferencias significativas, con un control de 97.31 a $99.08 \%$ de las poblaciones, mientras que el testigo presento una reducción significativamente menor, con una supervivencia de $87.16 \%$ ( $\mathrm{gl}=8$, $24 ; F=334.12 ; p<0.0001 ; \mathrm{R}^{2}=0.99, \mathrm{CV}=4.07$ ) (Cuadro 3). 
Los extractos vegetales de Nemaxxion XT Plus no permitieron que la densidad poblacional de $M$. incognita se incrementara, reduciéndose en un rango de 0.009 a 0.026 veces a la población inicial, principalmente en el tratamiento 3 (Nemaxxion XT Plus $6 \mathrm{~L} \mathrm{ha}^{-1}$ ), el cual resultó con mayor eficiencia con $98.93 \%$ (Cuadro 4).

Cuadro 4. Índice de incremento poblacional de Meloidogyne incognita y Pratylenchus sp., y eficiencia de los tratamientos evaluados para el control de nematodos.

\begin{tabular}{cccccc}
\hline \multirow{2}{*}{ Tratamientos } & \multicolumn{2}{c}{ Meloidogyne incognita } & & \multicolumn{2}{c}{ Pratylenchus sp. } \\
\cline { 2 - 3 } \cline { 5 - 6 } & Índice de incremento & Eficiencia (\%) & & Índice de incremento & Eficiencia (\%) \\
\hline 1 & 0.026 & 96.96 & & 0.125 & 90.34 \\
2 & 0.016 & 98.11 & & 0.121 & 90.62 \\
3 & 0.009 & 98.93 & & 0.067 & 94.82 \\
4 & 0.017 & 98.05 & & 0.122 & 90.6 \\
5 & 0.866 & 0 & & 1.294 & 0 \\
\hline
\end{tabular}

La aplicación de los tratamientos para el nematodo lesionador Pratylenchus sp., presentaron una efectividad de control significativa ( $p<0.05$ ) que fluctuó de 87.47 a $92.87 \%$ (Cuadro 3), tratamientos que redujeron la densidad de población en un rango de 0.067-0.125 veces la población inicial, destacando con mayor eficiencia $\left(94.82 \%\right.$ ) el tratamiento 3 (Nemaxxion XT Plus $6 \mathrm{~L} \mathrm{ha}^{-1}$ ) (Cuadro 4).

El tratamiento testigo sin control de nematodos, no presentó reducción en la población (Cuadro 3 ) $\left(\mathrm{gl}=8,24 ; F=416.54 ; p<0.0001 ; \mathrm{R}^{2}=0.99 ; \mathrm{CV}=4.35\right)$, con un incremento de la población de 1.294 veces (Cuadro 4), aumentando 13 nematodos más a la población de inicio (Cuadro 2).

Con base en el resultado, la utilización del producto Nemaxxion XT Plus a base de extractos vegetales y componentes orgánicos presento una alta eficiencia para el control de $M$. incognita y Pratylenchus sp., reduciendo la densidad de las poblaciones en el cultivo del cafeto; cuyo efecto es similar al producto organofosforado Nemacur ${ }^{\circledR} 400 \mathrm{CE}$, lo que sugiere que el producto de origen biológico puede ser utilizado como alternativa de control para estos nematodos.

Diversos autores han señalado la importancia y efectividad de utilizar alternativas ecológicas para el manejo de nematodos, principalmente extractos de plantas, lo que permite el desarrollo de una agricultura más rentable y no contaminante del ambiente (Parada y Guzmán, 1997; Dama, 2002; Jothi et al., 2004; Vinueza et al., 2006; Zakaria et al., 2013; Garrido et al., 2014).

\section{Conclusiones}

El Nemaxxion XT Plus es efectivo para el control del nematodo agallador Meloidogyne incognita y el nematodo lesionador Pratylenchus sp., en el cultivo del cafeto, además de que por sus características orgánicas e inocuas con el ambiente puede ser utilizado en cualquier sistema de producción agrícola, presentándose como alternativa para una agricultura más sustentable. 


\section{Literatura citada}

AMECAFÉ. 2016. Asociación Mexicana de la Cadena Productiva del Café AC. http://amecafe.org.mx/.

ANACAFÉ. 2017. Asociación Nacional del Café. Nematodos y su control. https://www.anacafe.org/glifos/index.php?title=Caficultura_ControlNematodos.

Baños, Y. S.; Concepción, A. B.; Lazo, R. C.; González, I. A. y Morejón, L. P. 2010. Efecto de enmiendas orgánicas y Trichoderma spp. en el manejo de Meloidogyne spp. Rev. Bras. Agroecol. 5(2):224-233.

Campos, V. P. and Villain, L. 2005. Nematode parasite of coffee and cocoa. In: plant parasitic nematodes in subtropical and tropical agriculture. Luc, M.; Sikora, R. A. y Bridje, J. (Ed.). CABI Publishing, Wellingford, UK. 529-579 pp.

Carneiro, R. M. D. G. and Cofcewicz, E. T. 2008. Taxonomy of coffee-parasitic root-knot nematodes, Meloidogyne spp. In: Plant-parasitic nematodes of coffee. Souza, R. M. (Ed.). Springer Science+Business Media B. V. Dordrecht, Netherlands. 87-122 pp.

Cepeda, S. M. 1995. Prácticas de nematología agrícola. Ed. Trillas, SA de CV México, DF. 109 p.

Cepeda, S. M. 2016. Nematología Agrícola. ${ }^{\text {da. }}$ reimpresión Ed. Trillas, SA de CV. México, DF. $304 \mathrm{p}$.

Dama, L. B. 2002. Effect of naturally occurring napthoquinones on root-knot nematode Meloidogyne javanica. Indian Phytopathology. 55(1):67-69.

Eisenback, D.; Hirschmann, J.; Sasser, H. and Triantaphyllou, A. 1981. Guide to the four most common species of root-knot nematodes (Meloidogyne spp.), with a pictorial key. The Departments of Plant pathology and Genetics North Carolina State University and The United States Agency for International Development. Raleigh, North Carolina. 48 p.

FAO. 2017. Organización de las Naciones Unidas para la Alimentación y la AgriculturaFAOSTAT. Disponible en: http://www.fao.org/faostat/es/\#data/QC.

Fe, A. M. 2002. Estrategias en el control y manejo de nematodos fitoparásitos. Ciencia y Medio Ambiente. 221-227 pp. http://digital.csic.es/bitstream/10261/128310/1/Estrategias\%20 en\%20el\%20control392\%28M\%c2\%aaF\%20Andr\%c3\%a9s\%29.pdf.

Garrido, C. F.; Cepeda, S. M.; Hernández, C. F. D.; Ochoa, F. Y. M.; Cerna, C. E. y Morales, A. D. M. 2014. Efectividad biológica de extractos de Carya illinoensis, para el control de Meloidogyne incognita. Rev. Mex. Cienc. Agric. 5(7):1317-1323.

Henderson, C. F. and Tilton, E. W. 1955. Tests with acaricides against brown wheat mite. J. Econ. Entomol. 48(2):157-161.

Hernández, J. A.; Cepeda, S. M.; Gallegos, M. G.; Chacón H. J. C.; Ordaz, S. S. y González R. A. 2015. Actividad nematicida de productos orgánicos comerciales, contra Ditylenchus dipsaci (Tylenchida: Anguinidae) bajo condiciones de laboratorio. Rev. Mex. Cienc. Agric. 11(Pub. Esp.):2247-2252.

ICO. 2017. International Coffee Organization. Estadísticas comerciales del café. http://www.ico.org/ES/trade_statisticsc.asp.

Inomoto, M.; Oliveira, M.; Mazzafera, P. and Goncalves, W. 1998. Effects of Pratylenchus brachyurus and P. coffeae on seedlings of Coffea arabica. J. Nematol. 30(3):362-367.

Jothi, G.; Sundara B. R.; Ramakrishnan, S. and Rajendran, G. 2004. Management of root lesion nematode, Pratylenchus delattrei in crossandra using oil cakes. Bioresour. Technol. 93(3):257-259. 
Oliveira, C.; Inomoto, M.; Vieira, A. y Monteiro, A. 1999. Efeito de densidades populacionais de Pratylenchus brachyurus no crescimiento de plántulas de Coffea arabica cv. Mundo novo e C. canephora cv. Apoatá. Nematropica. 29(2):215-221.

Parada, R. Y. y Guzmán, R. F. 1997. Evaluación de extractos botánicos contra el nematodo Meloidogyne incognita en frijol (Phaseolus vulgaris). Agron. Mesoam. 8(1):108-114.

Pérez, P. J. R. y Díaz, C. S. 2000. El café: bebida que conquistó al mundo. Universidad Autónoma de Chapingo. Centro Regional Universitario Oriente, Programa de Investigación y Desarrollo en Regiones Cafetaleras. México. 151 p.

Perry, R. N. and Wright, D. J. 1998. The physiology and biochemistry of free-living and plantparasitic nematodes. CABI Publishing. Wallingford, U. K. 438 p.

SAGARPA-SIAP. 2017. Secretaría de Agricultura, Ganadería, Desarrollo Rural, Pesca y Alimentación. Servicio de Información Agroalimentaria y Pesquera-Anuario Estadístico de la Producción Agrícola. http://infosiap.siap.gob.mx/aagricola_siap_gb/icultivo/index.jsp.

SAS. Institute. 2002. The SAS System for Windows, Release 9.0. SAS, Institute, Cary NC, USA.

Seinhorst, J. W. 1970. Dynamic of populations of plant parasitic nematodes. Annu. Rev. Phytopathol. 8(1):131-156.

Souza, R. M. and Bressan-Smith, R. 2008. Coffee-Associated Meloidogyne spp.-ecology and Interaction with Plants. pp. 123-147. In: Souza, R. M. (Ed.). Plant-parasitic nematodes of coffee. Springer Science+Business Media B. V. Dordrecht, Netherlands. 340 p.

Taylor, D. P. and Netscher C. 1974. An improved technique for preparing perineal patterns of Meloidogyne spp. Nematológica. 20(2):268-269.

Villain, L. 2008. Economic importance, epidemiology and management of Pratylenchus sp. in coffee plantations. pp. 65-84. In: Souza, R. M. (Ed.). Plant-parasitic nematodes of coffee. Springer Science+Business Media B. V. Dordrecht, Netherlands. 340 p.

Vinueza, P. S. M.; Crozzoli, R. y Perichi, G. 2006. Evaluación in vitro de extractos acuosos de plantas para el control del nematodo agallador Meloidogyne incognita. Fitopatol. Venezol. 19(2):26-31.

Zakaria, H. M.; Kassab, A. S.; Shamseldean, M. M. and Oraby, M. M. 2013. Controlling the rootknot nematode, Meloidogyne incognita in cucumber plants using some soil bioagents and some amendments under simulated field conditions. Ann. Agric. Sci. 58(1):77-82. 